\title{
REALISATION OF AWGN CHANNEL EMULATION MODULES UNDER SISO AND SIMO ENVIRONMENTS FOR 4G LTE SYSTEMS
}

\author{
Dr. R. Shantha Selva Kumari ${ }^{1}$ and M. Aarti Meena ${ }^{2}$ \\ ${ }^{1}$ Department of Electronics and Communication Engineering, Mepco Schlenk \\ Engineering College, Sivakasi \\ ${ }^{2}$ Department of Electronics and Communication Engineering, Mepco Schlenk \\ Engineering College, Sivakasi
}

\begin{abstract}
The testing of a wireless transmitter and receiver in the real-world channel is tedious. So, a channel emulator using FPGA helps in the testing of transmitter and receiver by providing a test environment that simulates a real-world wireless channel. Since FPGAs are flexible, cheap and reconfigurable, they are used in designing an AWGN channel emulator for 4G LTE for Single Input Single Output (SISO) and Single Input Multiple Output (SIMO) environments. In this paper, three basic modules: transmitter, channel estimation and receiver modules are synthesized. In the transmitter module, the input data is 64QAM modulated and transmitted into the channel. In the channel estimation module, the transmitter data gets multiplied with the channel coefficients and then added with the noise present in the channel. In the receiver module, the data is detected using MMSE estimation. These are implemented in Virtex-5 device using PlanAhead tool and the Resource and Power Estimations are discussed.
\end{abstract}

\section{KEYWORDS}

AWGN, CFI, Channel coefficients, Channel estimation, MMSE, Pre-computed values, QAM modulation.

\section{INTRODUCTION}

Channel emulators are of great significance in the testing and verification of wireless communication systems. Design and testing of wireless communication systems using simple tools becomes necessary since the present equipment are not accurate and they cost more. Due to the different characteristics of FPGA like, easy integrated mapping, fast processing, low cost and reconfiguration, the synthesis of channel emulation could be done in an effective way.

Long Term Evolution (LTE) of UMTS (Universal Mobile Telecommunication Systems) is a Fourth Generation wireless broadband technology. The main advantages of LTE are high data rate and low latency with reduced cost, when compared with its predecessor GSM.

In this paper, a channel emulator for AWGN (Additive White Gaussian Noise) is designed for SISO and SIMO environments for LTE systems. They are implemented using PlanAhead tool and Virtex-5 device. The channel is estimated on the basis of BER. Bit Error Rate is defined as the ratio of the error bits to the number of bits transmitted. As the Signal-to-noise ratio increases, BER decreases and vice versa. 


\section{SYSTEM MODEL}

In the transmitter side, it can be observed that the input data is fed into a modulator block. The input data given is four sets of 36 - bits. Those are modulated by means of a 64-QAM modulator. After modulation, the signal is transmitted into the channel, so that it can travel long distances. After modulation, there will be 6 sets of 16 - bit data, sent into the channel.

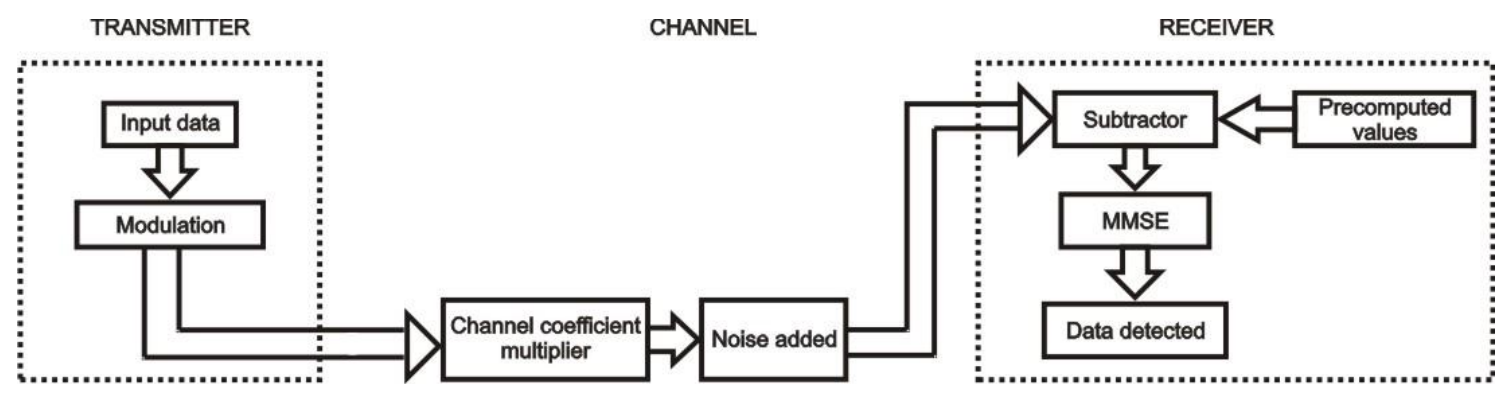

Figure 1. System Model of Channel Emulation process

In the channel, the 6 sets of 16 - bit modulated data are multiplied with the 16 sets of 16 - bit channel coefficients which are already present in the channel. Then the resulting data will be added with the noise present in the channel. Channel coefficients are the discrete-time impulse response of the channel. Ideally, one transmitting symbol gives an output only in one symbol time without interfering with other symbols. The 16 sets of 16 - bit data which are already present in the channel are of random in nature. These values are not directly added with the multiplied values. Instead, the 6 sets of 16 - bit noise are multiplied with the variance.

The formula for variance is given by,

$$
\text { Variance }=1 \text { sqrt } 2 * 10^{\wedge}-\mathrm{SNR} 20
$$

The noise value after getting multiplied with the variance gets added with the multiplied values. This value is received by the receiver.

In the receiver side, the data from the channel is received and it is subtracted from a set of precomputed values. The pre-computed values are the product of the 6 sets of 16 - bit QAM modulated signal with the 6 sets of 16 - bit channel coefficients. It does not contain the noise which is present in the channel. After subtraction, the resultant value is given as input to the Minimum Mean Square Error estimator (MMSE), to find the minimum value. That minimum value is called as Control Format Indicator (CFI). The data corresponding to the CFI is given as the output by the receiver. The input data is a complex one and hence the output will also be a complex one.

The complex-valued output at the k-th receiving antenna is modelled as,

$$
\mathrm{y}_{\mathrm{k}}=\mathrm{h}_{\mathrm{k}} \circ \mathrm{d}(\mathrm{n})+\mathrm{u}_{\mathrm{k}}, \quad \mathrm{k}=1,2, \ldots \mathrm{K}
$$

where, $y$ is the received signal, $h$ is the channel coefficient and $u$ is the noise.

The minimum value will be calculated by, 
which simplifies to

$$
\mathrm{CFI}=\min _{\mathrm{m}=1,2,3} \quad \underset{\mathrm{k}=1}{\mathrm{~K}} \mathrm{y}_{\mathrm{k}}-\mathrm{h}_{\mathrm{k}} \circ \mathrm{d}^{\mathrm{m}}{ }^{2}
$$

$$
\mathrm{CFI}=\arg _{\mathrm{m}=1,2,3} \mathbf{z}^{(\mathrm{m})}
$$

where the soft outputs are given by,

$$
\mathbf{z}^{\mathrm{m}}=\underset{\mathrm{k}=1}{\mathrm{~K}} \mathbf{z}_{\mathrm{k}}^{\mathrm{m}} \text { for } \mathrm{m}=1,2,3
$$

where $\mathrm{z}_{\mathrm{k}}^{\mathrm{m}}=\operatorname{Re} \mathrm{y}_{\mathrm{k}}{ }^{\circ} \mathrm{h}_{\mathrm{k}}^{*}, \mathrm{~d}^{\mathrm{m}} \quad$ for $\mathrm{m}=1,2,3$.

By means of these equations [1], the minimum CFI value is calculated. Then the output retrieved by the receiver is compared with the original data. For every correct value, a counter will count the values. Based on this, BER graph is drawn.

In the case of SISO, there will be a single receiving antenna, whereas in the case of SIMO, there will be two receiving antennae.

\section{ARCHITECTURE OF SISO}

The architecture of SISO consists of only one transmitting antenna and one receiving antenna as shown in Figure 2.

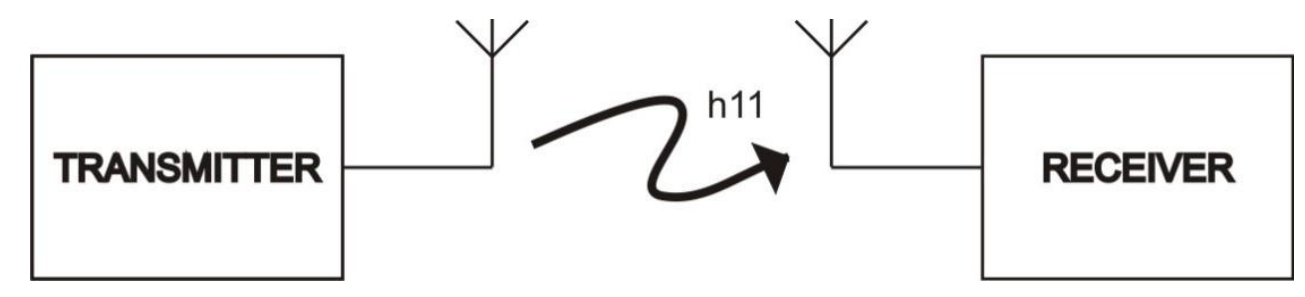

Figure 2. Basic block diagram of SISO 


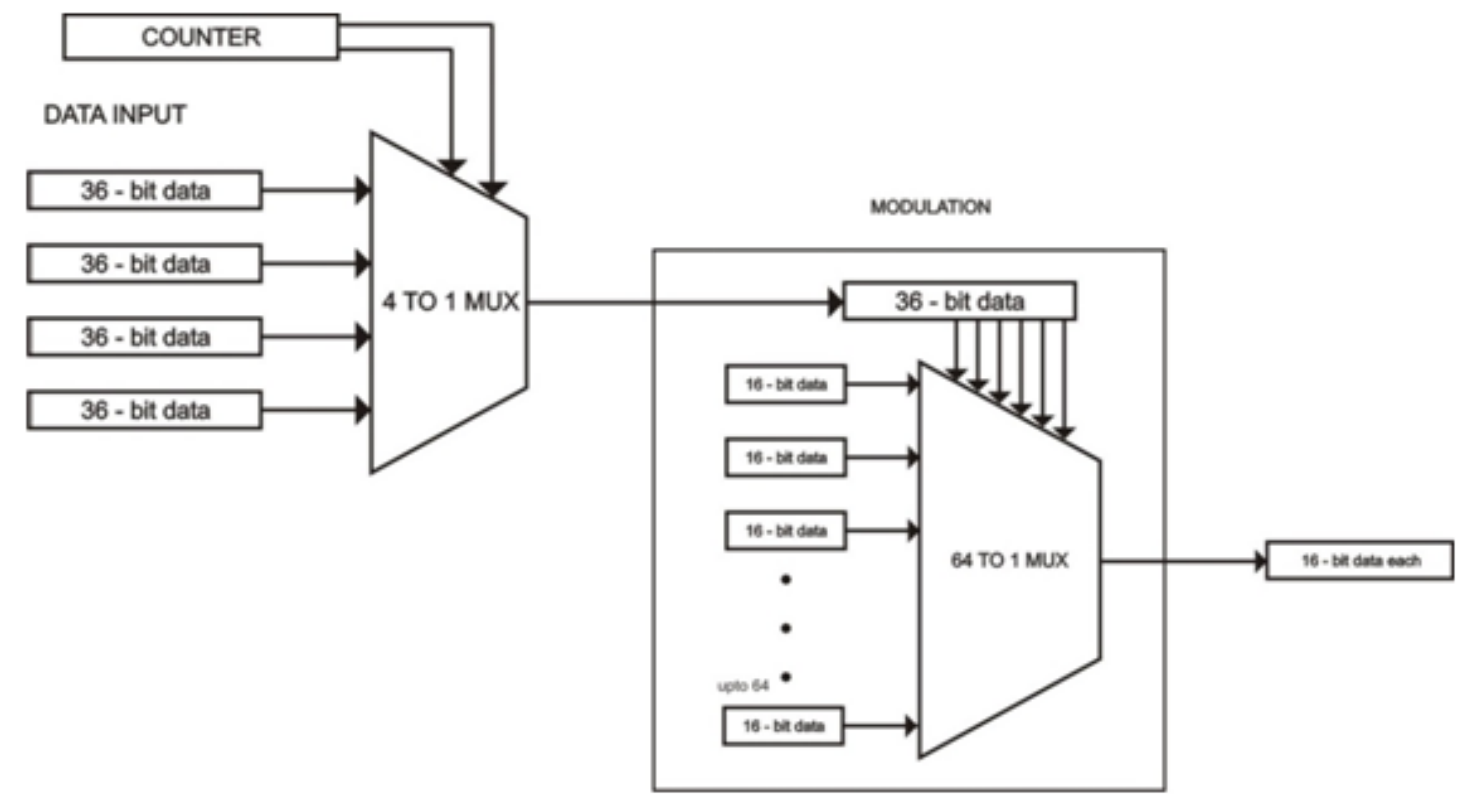

Figure 3. Transmitter side architecture of SISO

From the Figure 3, it is observed that the input given is four sets of 36 - bit data (18 - bit real, 18 bit imaginary). The output data are given as input to a 4 to 1 MUX. The 2 - bit control input to the MUX are given by a 2 - bit counter. The counter will count for every clock cycle. So for every clock pulse a 36 - bit data will be sent out of the MUX. The next block is a 64-QAM Modulator block. The QAM modulator block consists of four sets of 16 - bit given as input to a 4 to 1 MUX. Each 2 - bit data from the received 36 - bit will act as the control input to the MUX. This is performed by means of a 2 - bit shifter. Based on the values of the 2 - bit data, a 16 - bit data will be given as the output from the modulator. The output will be the QAM modulated signal. Since from a 36 - bit data, modulation takes place for every 2 - bits, the output will be 6 sets of 16 - bit QAM modulated signal. Thus for every clock pulse the output will be 6 sets of 16 - bit QAM modulated signal. 
International Journal of Wireless \& Mobile Networks (IJWMN) Vol. 6, No. 6, December 2014

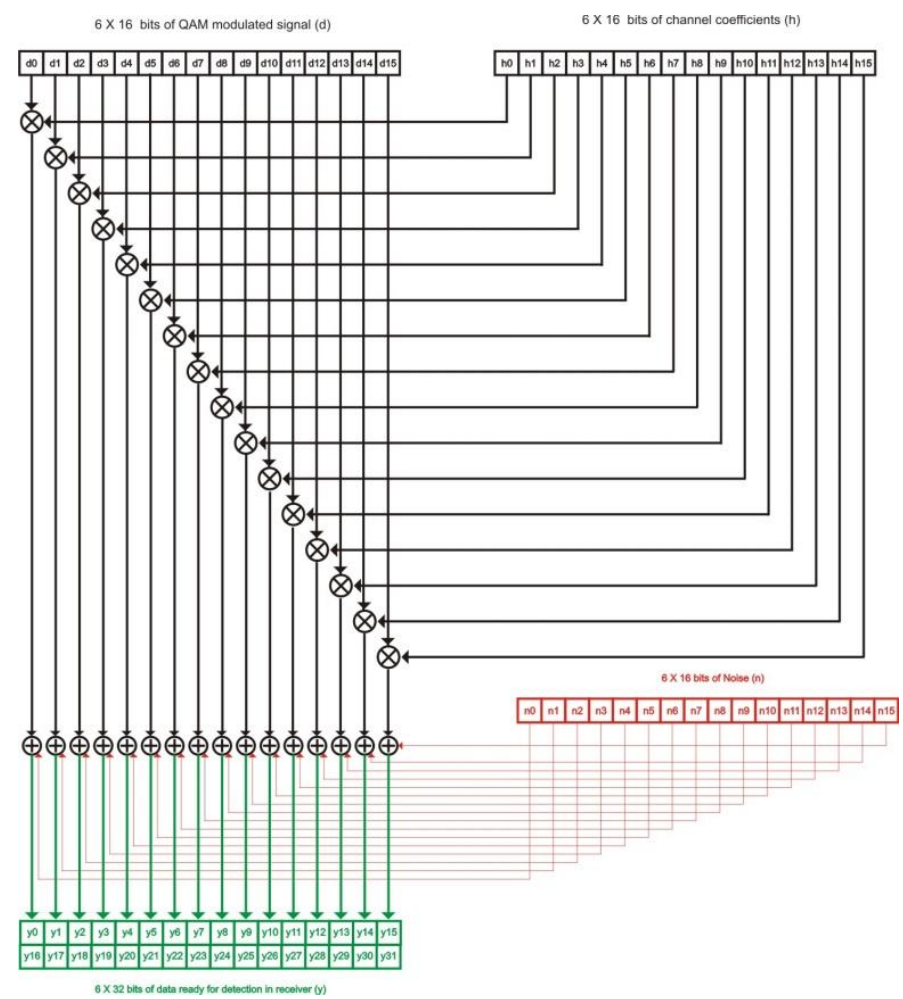

Figure 4. Channel Estimation architecture of SISO

From the Figure 4, it is can be seen that the output from the transmitter side, which is the $6 \times 16$ bits QAM modulated signal, is multiplied with the $6 \times 16$ bits of channel coefficients [5]. Then the multiplied value is added with the $6 \times 16$ bits of noise which is present in the channel. The noise which gets added will be a product of the variance and the noise. The resulting output will be $6 \times 32$ bits of data which is to be received by the receiver. 


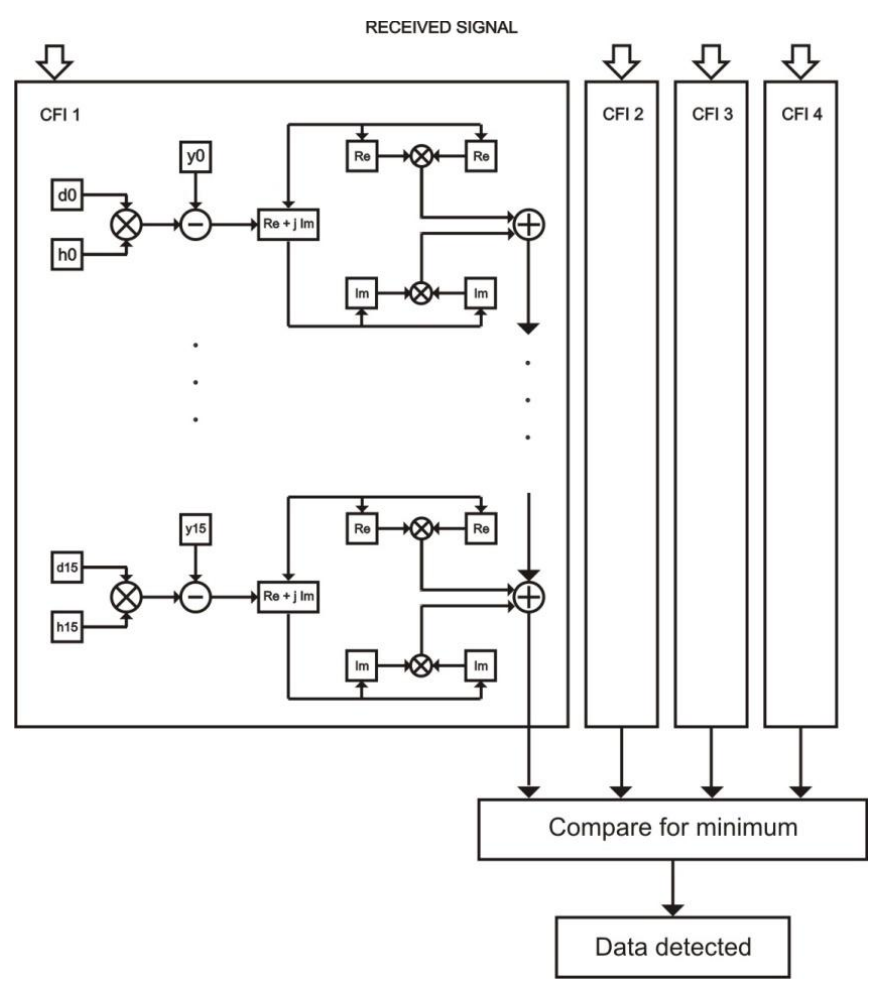

Figure 5. Receiver side architecture of SISO

From the Figure 5, it is observed that in the receiver side, pre-computed data will be present. The pre-computed data is the product of the QAM modulated signal and the channel coefficients value. From the received data, the pre-computed values are subtracted. From the obtained result, real parts and imaginary parts are separately squared and added with each other. This is performed for all the 6 sets of 16 bit data. This is for a single clock pulse. Similarly for all the clock cycles, the values are calculated. Since there are four sets of 36 - bit data, after four clock cycles only the output can be obtained [6]. Hence there will be a delay of four clock cycles. After four clock cycles, there will be four sets of values. These values are compared for minimum, by means of Minimum Mean Square Error (MMSE) estimator algorithm. From this, the minimum value is detected and it is given as the detected output.

\section{ArChitecture OF SIMO}

The architecture of SIMO consists of only one transmitting antenna and many receiving antennae. In this paper, a $1 \times 2$ SIMO is considered (i.e.) one transmitting antenna and two receiving antennae, as shown in Figure 6.

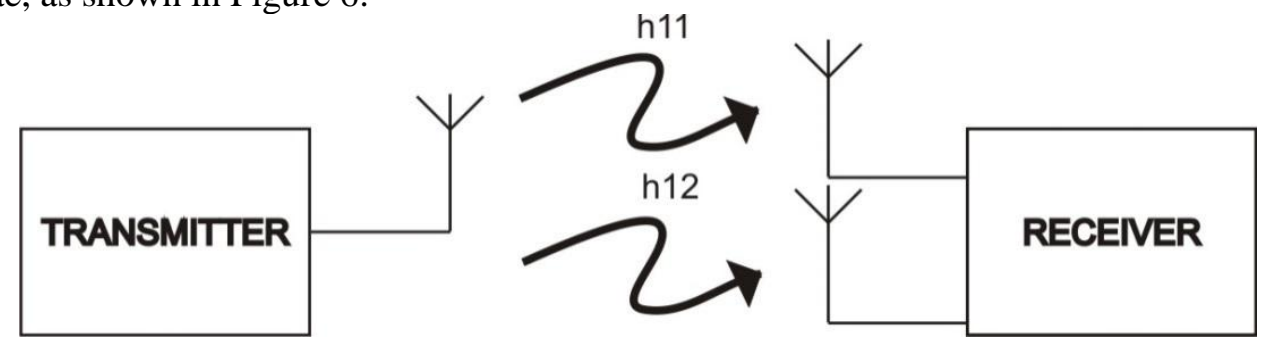

Figure 6. Basic block diagram of SIMO 
The transmitter block of SIMO is similar to that of SISO. But the channel estimation and receiver blocks will vary.

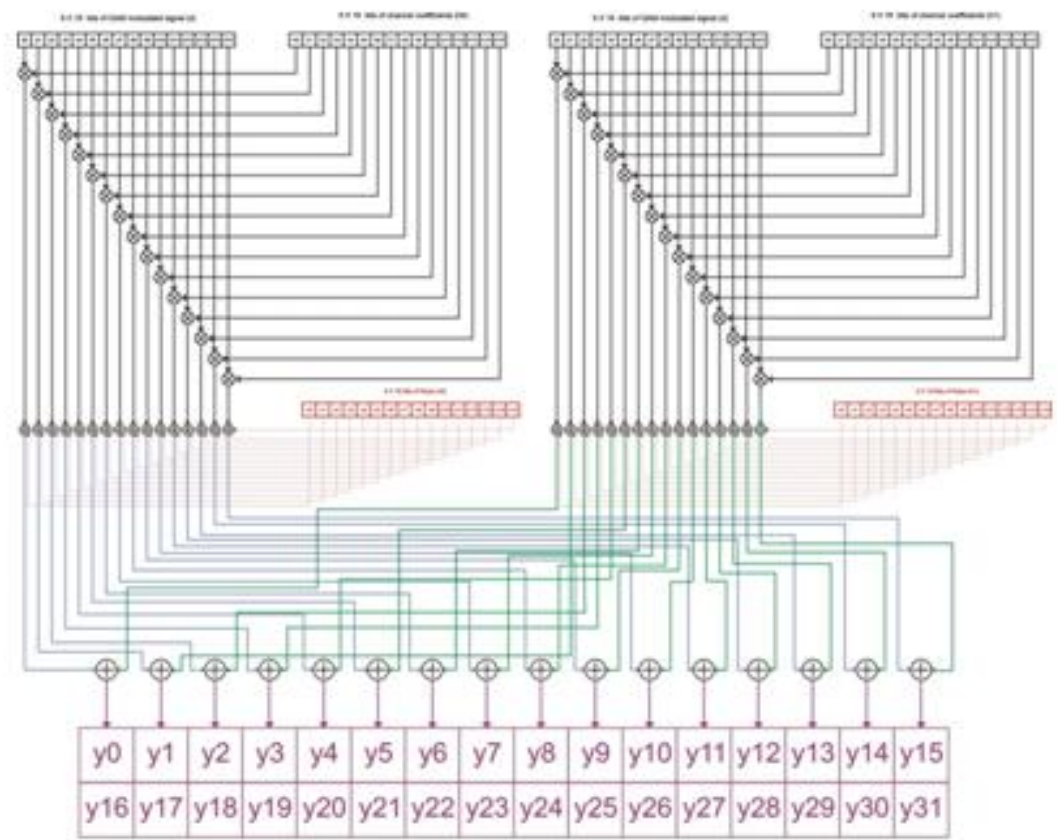

$6 \times 32$ bits of data ready for detection in receiver $(y)$

Figure 7. Channel Estimation architecture of SIMO

From the Figure 7, it is observed that the output from the transmitter side, which is the $6 \times 16$ bits QAM modulated signal, is multiplied with two sets of $6 \times 16$ bits of channel coefficients, which are $\mathrm{h} 0$ and $\mathrm{h} 1$. After that, there will be two sets of multiplied values. These values are added with two sets $6 \times 16$ bits of noise, which are $n 0$ and $n 1$, which is present in the channel. The noise which gets added will be a product of the variance and the noise. Then the two sets of values are added with each other. The resulting output will be $6 \times 16$ bits of data which is to be received by the receiver [5]. 


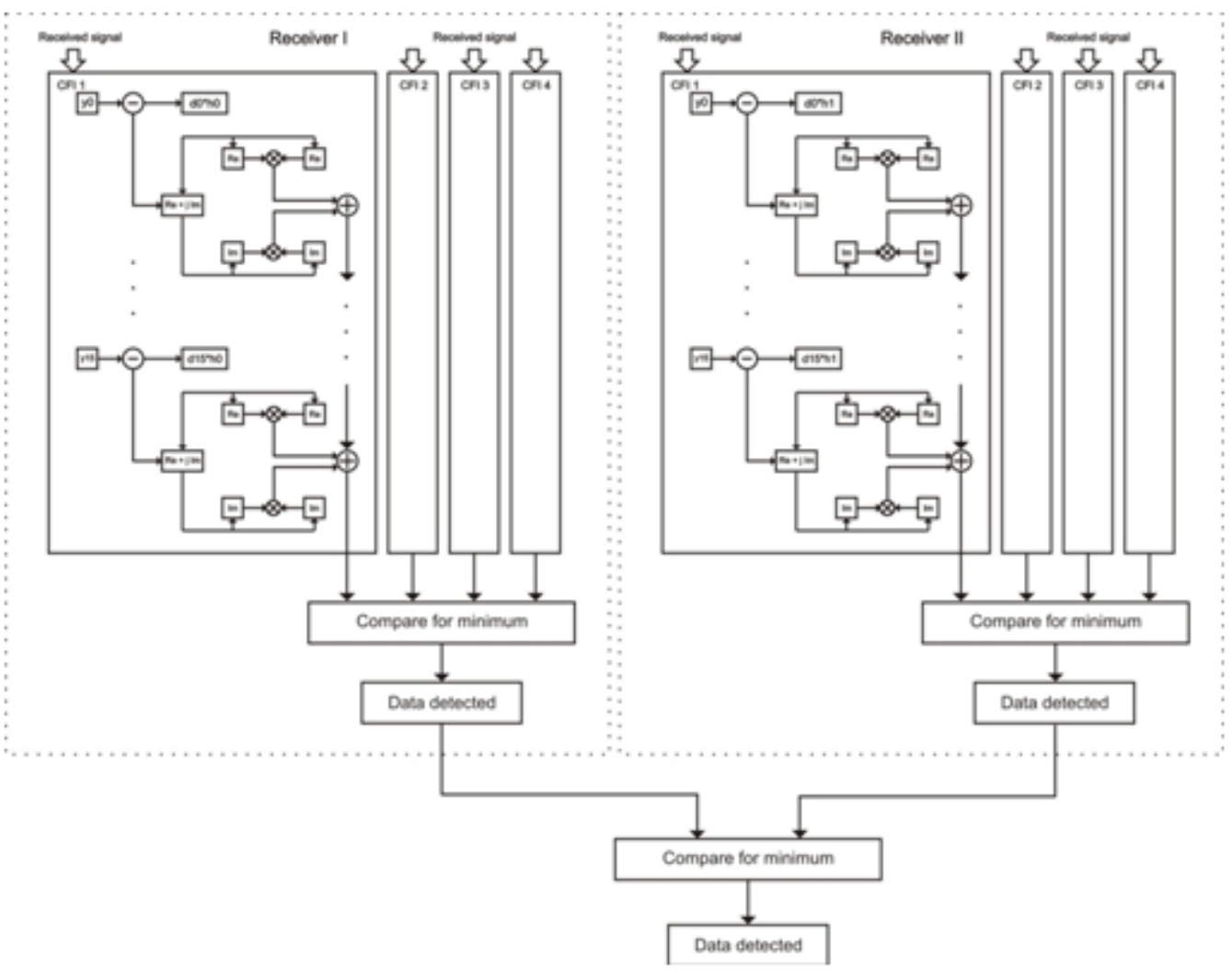

Figure 8. Receiver side architecture of SIMO

In general, in the receiver side, pre-computed data will be present. In the Figure 8, there are two receivers. So, Receiver I will contain pre-computed data which is the product of the QAM modulated signal and the channel coefficients value of h0. In Receiver II, the pre-computed data is the product of the QAM modulated signal and the channel coefficients value of $h 1$. But in both the receivers, the received data will be the same [6].

In each receiver, from the received data, the pre-computed values are subtracted. From the obtained result, real parts and imaginary parts are separately squared and added with each other. This is performed for all the 6 sets of 16 bit data. This is for a single clock pulse. Similarly for all the clock cycles, the values are calculated. Since there are four sets of 36 - bit data, after four clock cycles only the output can be obtained. Hence there will be a delay of four clock cycles.

After four clock cycles, there will be four sets of values, the values of CFI. These values are compared for minimum, by means of Minimum Mean Square Error (MMSE) estimator algorithm. From this, the minimum value is detected and it is given as the detected output. All these processes are executed separately for both the receivers. Finally from the detected output value from both the receivers, the minimum value is calculated. That value is considered as the original data, which is transmitted by the transmitter. 


\section{RESUlTS AND DisCUSSIONS}

Simulation is performed in Modelsim and implementation is accomplished by PlanAhead tool in Virtex-5 device.

\subsection{Simulation Result}

\subsubsection{SISO}

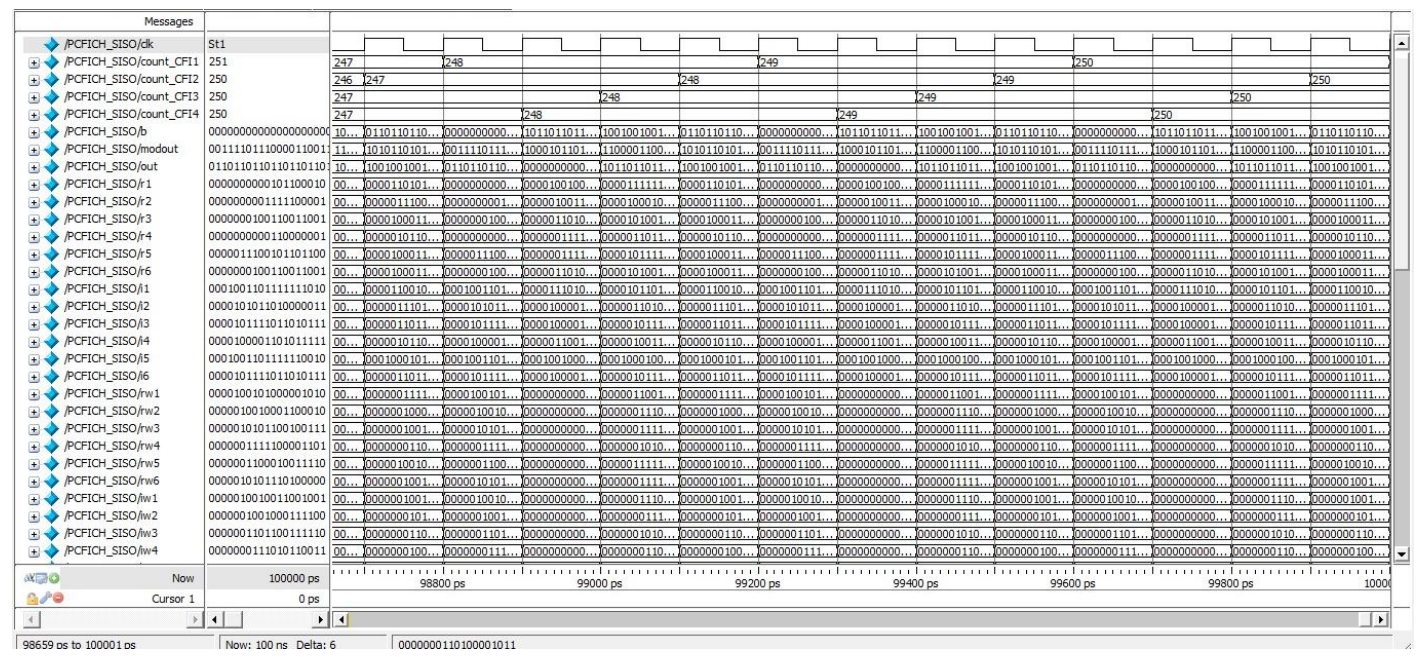

Figure 9. Simulation Result of SISO

From the Figure 9, it is observed that "clk" (clock) is the input given. The outputs count_CFI 1, count_CFI 2, count_CFI 3,count_CFI 4 are the count values which indicate the number of times the original data retrieved at the receiver is equal to the input data given. The Figure 9 indicates the simulation result for a case with variance 0 . If the value of variance is 0 , then there will be no noise and hence it is the ideal case. So there will be perfect reception of the transmitted signal at the receiver side. However, there will be a delay of four clock pulses and hence each output is counted after four clock pulses. So in the above case, for an input of 1000 clock pulses, the four sets of 36 - bit input data are retrieved 250 times in the Receiver.

\subsubsection{SIMO}

From the Figure 10, it is observed that "clk" (clock) is the input given. The outputs count1_CFI 1, count1_CFI 2, count1_CFI 3, count1_CFI 4 are the count values which indicate the number of times the original data is retrieved at the receiving end in Receiver $\mathrm{I}$ is equal to the input data given in the transmitting end. Similarly, the outputs count2_CFI 1, count2_CFI2, count2_CFI3, count2_CFI4 are the count values which indicate the number of times the original data retrieved at the receiving end in Receiver II is equal to the input data given in the transmitting end. 
International Journal of Wireless \& Mobile Networks (IJWMN) Vol. 6, No. 6, December 2014

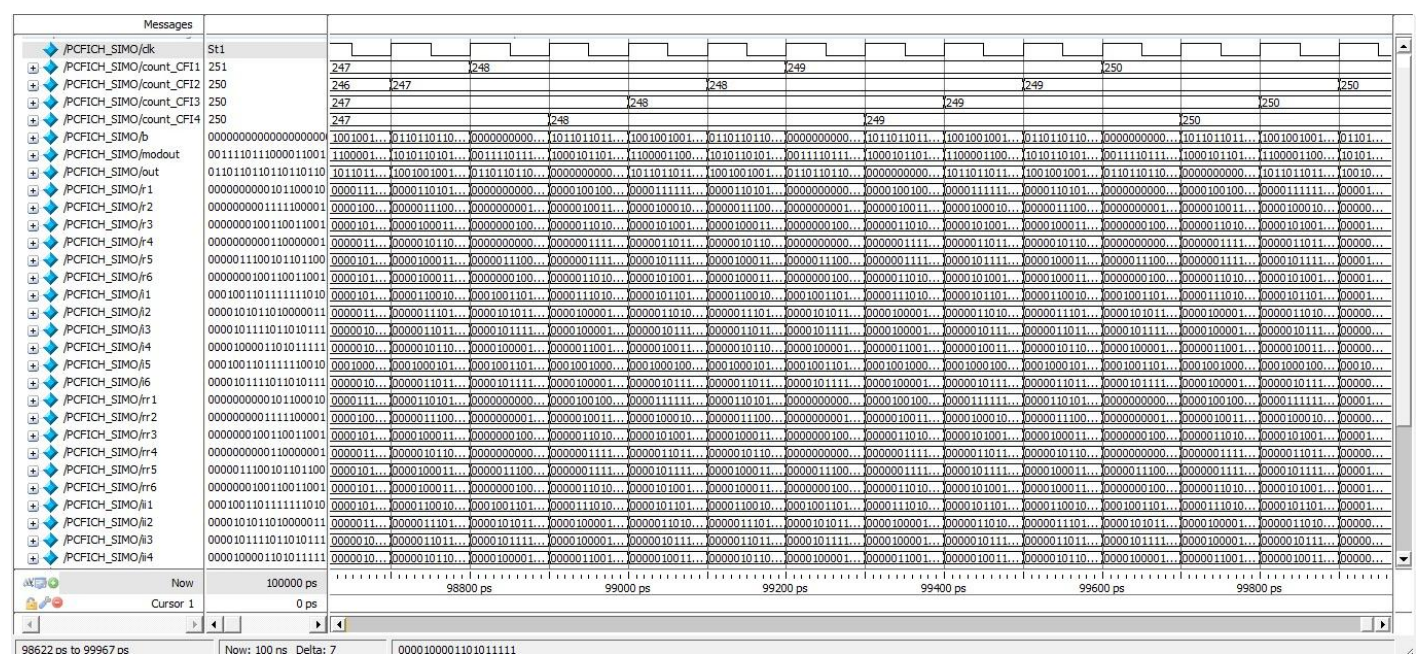

Figure 10. Simulation Result of SIMO

The Figure 10 indicates the simulation result for a case with variance 0 . If the value of variance is 0 , then there will be no noise and hence it is the ideal case. So there will be perfect reception of the transmitted signal at the receiver side. However, there will be a delay of four clock pulses and hence each output is counted after four clock pulses. So in the above case, for an input of 1000 clock pulses, the four sets of 36 - bit input data are retrieved 250 times in both the Receiver I and Receiver II.

\subsection{FPGA Editor}

In the FPGA Editor, the programmable elements are called Configurable Logic Blocks (CLB). A CLB contains LUTs (Look Up Table) and (Flip Flops) FFs/Latches. The LUTs and FFs are organized in Slices.

\subsubsection{SISO}

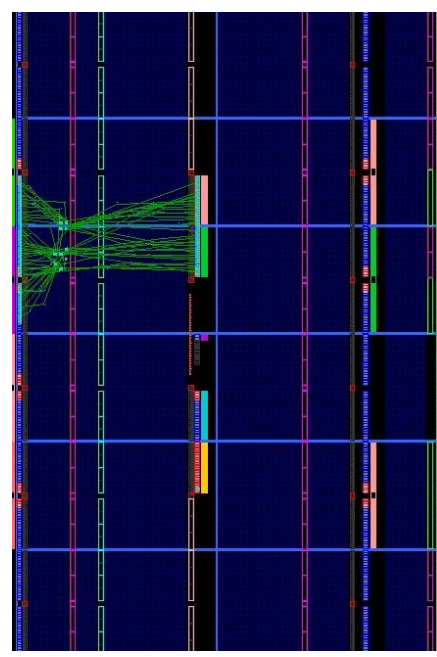

Figure 11. FPGA Editor of Transmitter

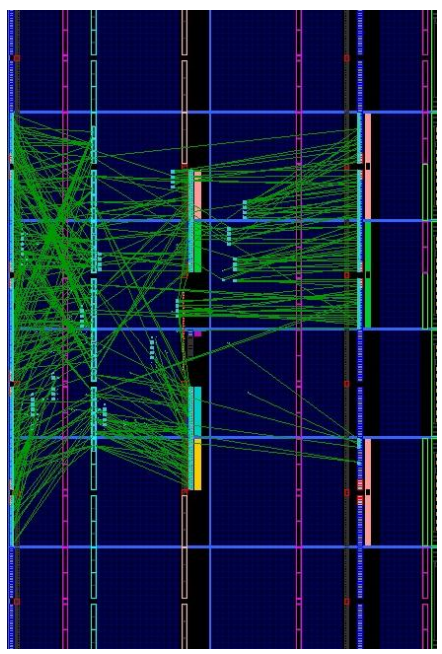

Figure 12. FPGA Editor of Channel

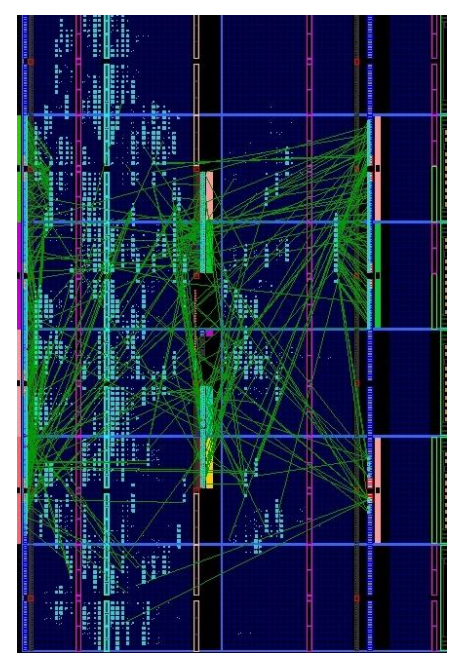

Figure 13. FPGA Editor of Receiver 
From the Figure 11, 12 and 13, it can be observed that there are various connecting wires between different LUTs and FFs, which are in green colour. These are the input and output connecting wires.

\subsubsection{SIMO}

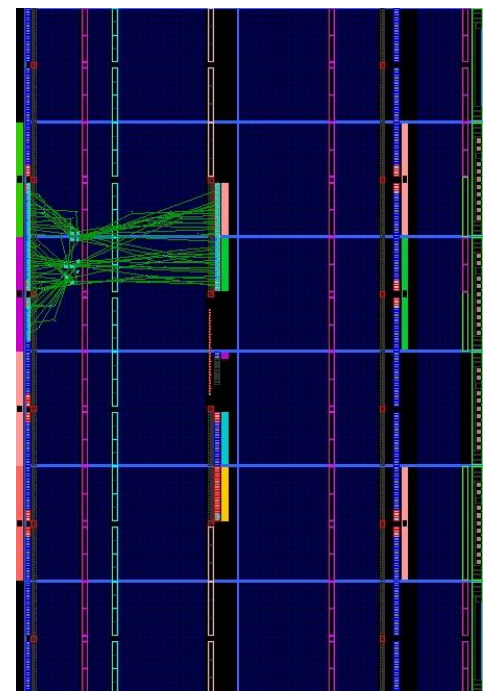

Figure 14. FPGA Editor of Transmitter

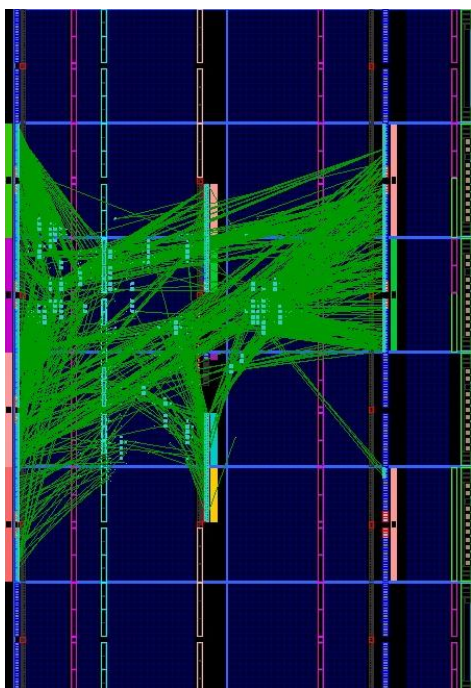

Figure 15. FPGA Editor of Channel

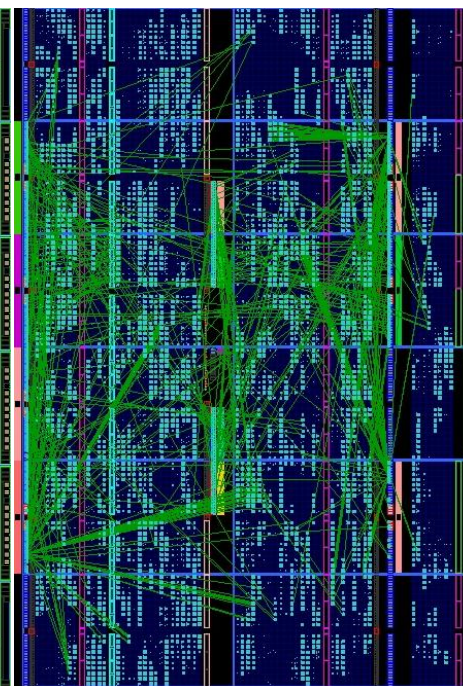

Figure 16. FPGA Editor of Receiver

From the Figure 14, 15 and 16, it can be observed that there are various connecting wires between different LUTs and FFs, which are in green colour. These are the input and output connecting wires.

\subsection{RTL Schematic}

RTL schematic is a representation of the pre-optimized design in terms of generic symbols, such as adders, multipliers, counters, AND gates, and OR gates.

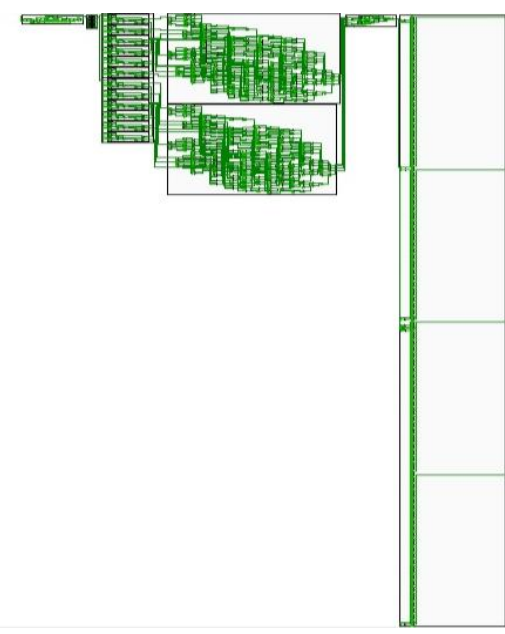

Figure 17. RTL Schematic of SISO

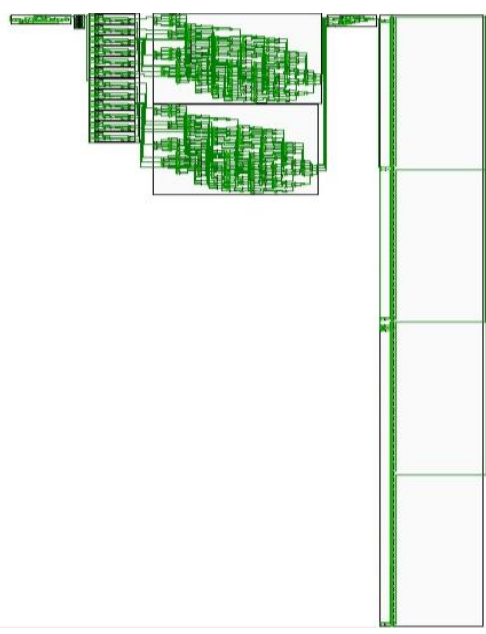

Figure 18. RTL Schematic of SIMO 
Figure 17 and 18 shows the RTL Schematic of SISO and SIMO respectively.

\subsection{Resource Estimation}

Resource estimation indicates the amount of resources that got utilized by the device. In general, it indicates the number of Registers, LUT, Slice, I/O, Buffers utilized.

\subsubsection{SISO}

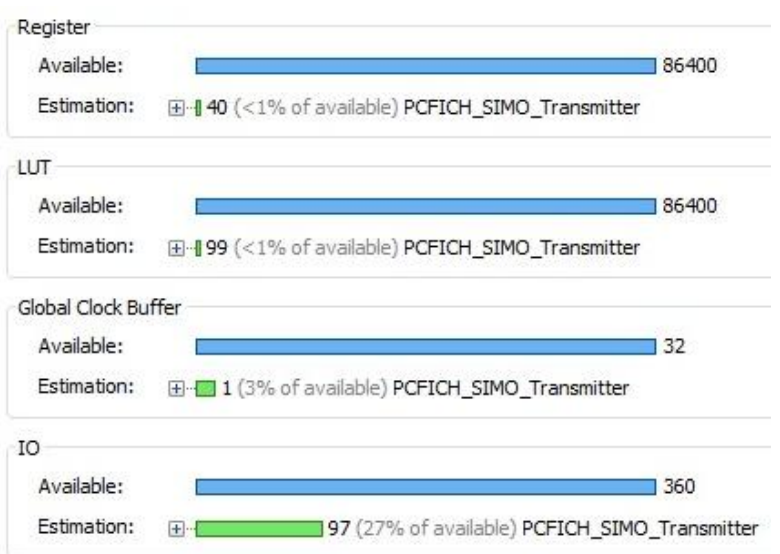

Figure 19. Resource Estimation of Transmitter

From the Figure 19, it can be seen that in the transmitter, the percentage of Registers consumed is $1 \%$, LUT is $1 \%$ and $\mathrm{IO}$ is $27 \%$.

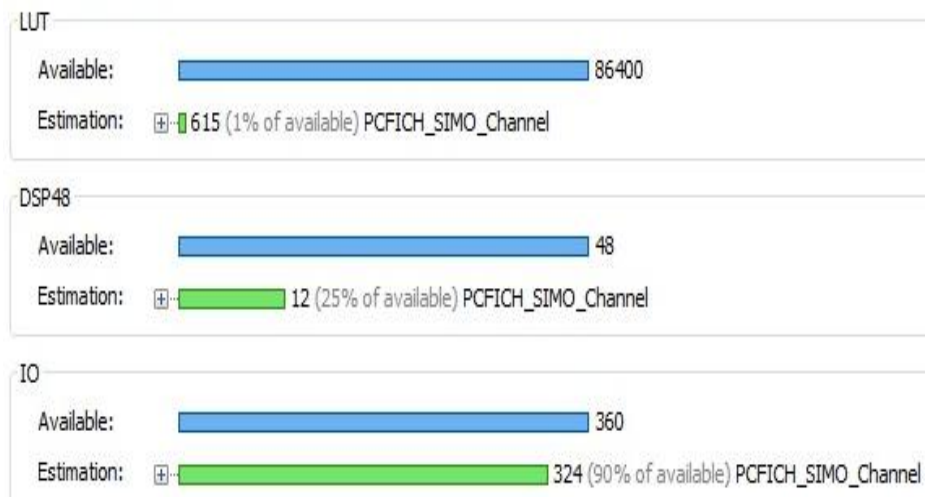

Figure 20. Resource Estimation of Channel

From the Figure 20, it is observed that in the channel, the percentage of LUT consumed is $1 \%$, Slice is $25 \%$ and $\mathrm{IO}$ is $90 \%$. 
International Journal of Wireless \& Mobile Networks (IJWMN) Vol. 6, No. 6, December 2014

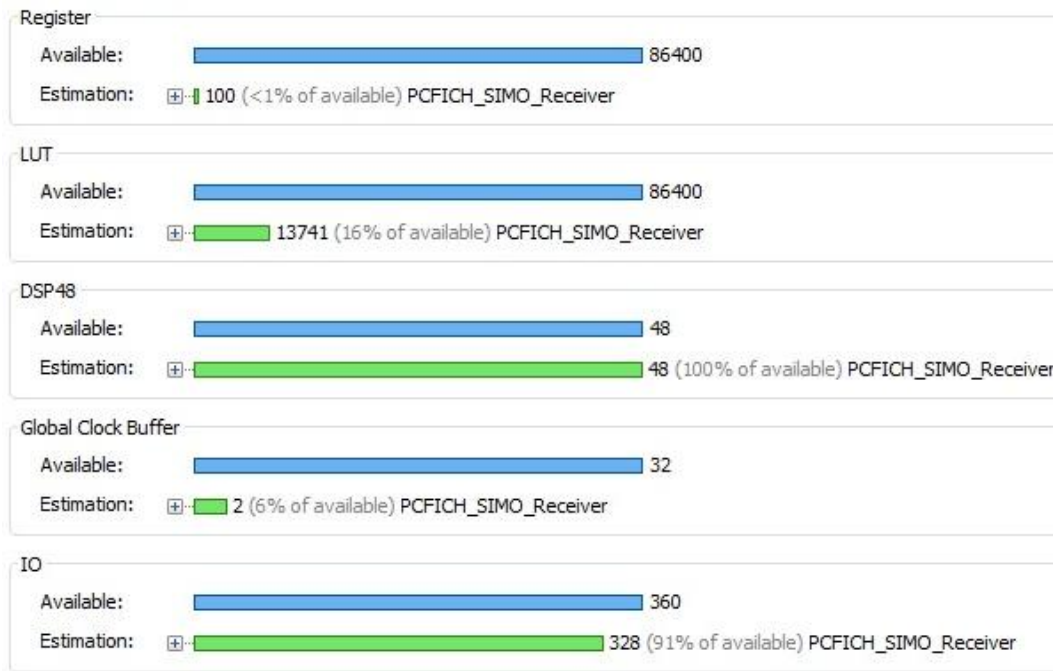

Figure 21. Resource Estimation of Receiver

From the Figure 21, it can be observed that in the receiver side, the percentage of Registers consumed is $1 \%$, LUT is $16 \%$ and IO is $91 \%$.

\subsubsection{SIMO}

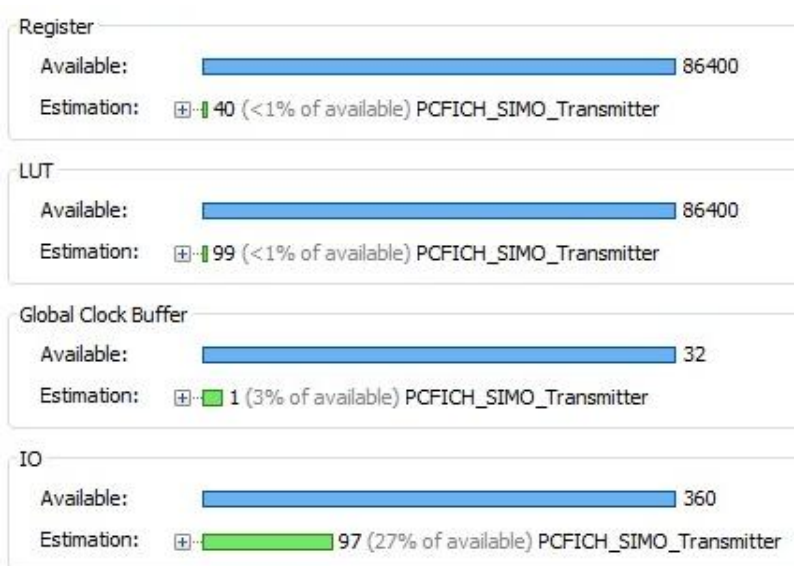

Figure 22. Resource Estimation of Transmitter

From the Figure 22, it can be observed that in the transmitter, the percentage of Registers consumed is $1 \%$, LUT is $1 \%$, Slice is $3 \%$ and IO is $27 \%$. 
International Journal of Wireless \& Mobile Networks (IJWMN) Vol. 6, No. 6, December 2014

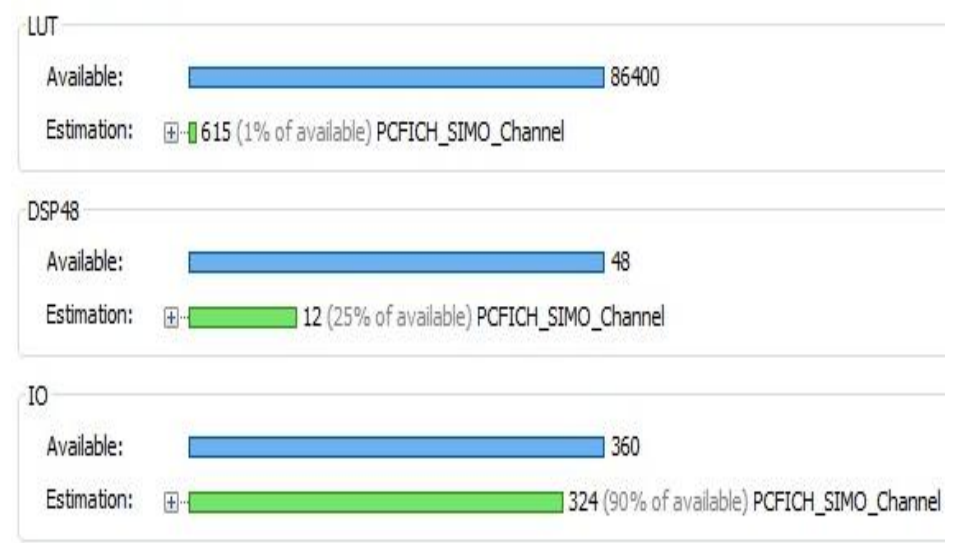

Figure 23. Resource Estimation of Channel

From the Figure 23, it is seen that in the channel, the percentage of Registers consumed is $1 \%$, LUT is $1 \%$, Slice is $25 \%$ and IO is $90 \%$.

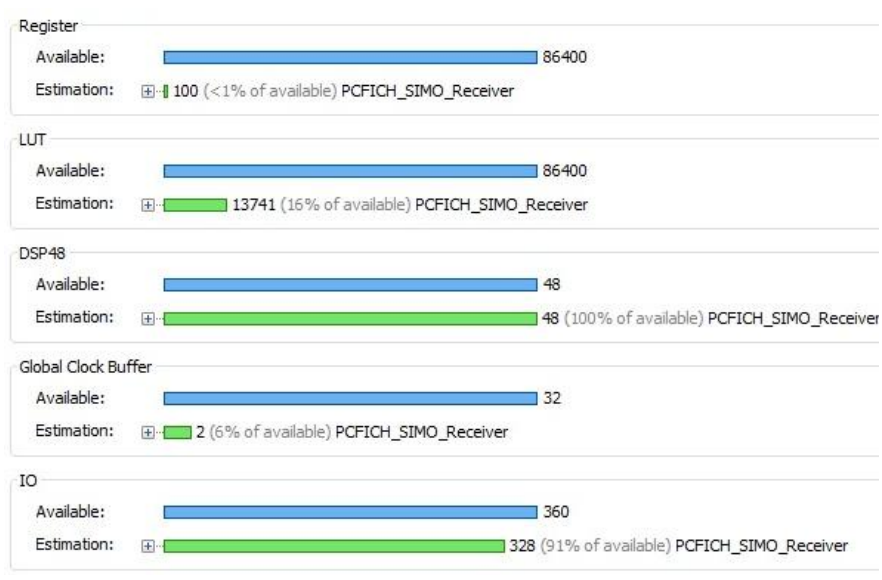

Figure 24. Resource Estimation of Receiver

From the Figure 24, it can be observed that in the receiver side, the percentage of Registers consumed is $1 \%$, LUT is $16 \%$ and IO is $91 \%$.

\subsection{Power Estimation}

Power estimation considers the design's resource usage, toggle rates, I/O loading, and many other factors and it combines them with the device models to calculate the estimated power.

There are three types of power estimated in Xilinx:

- I/O Power - It is the power consumed due to external switching.

- Core Dynamic Power - It is the power consumed due to internal switching.

- Device Static Power - It is the power consumed when the device is powered up without programming the used logic. The main contributor of this is the junction temperature. 


\subsubsection{SISO}

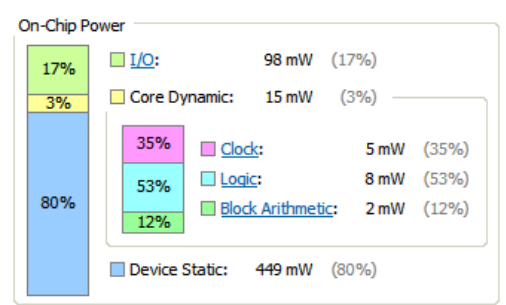

Figure 25. Power Estimation of Transmitter

From the Figure 25, it can be observed that the power consumed by I/O is $98 \mathrm{~mW}$, Core Dynamic is $15 \mathrm{~mW}$ and Device Static is $449 \mathrm{~mW}$ in the transmitter.

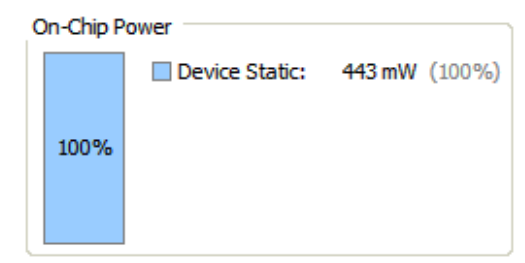

Figure 26. Power Estimation of Channel

From the Figure 26, it is observed that the power consumed by Device Static is $443 \mathrm{~mW}$ in the channel.

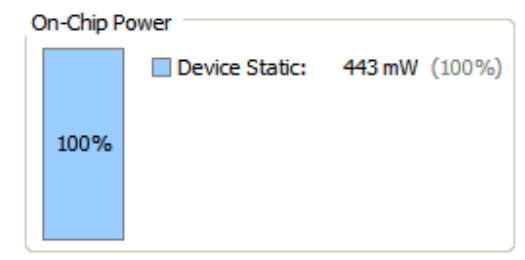

Figure 27. Power Estimation of Receiver

Similarly, from the Figure 27, it can be observed that the power consumed by Device Static is $443 \mathrm{~mW}$ in the receiver.

\subsubsection{SIMO}

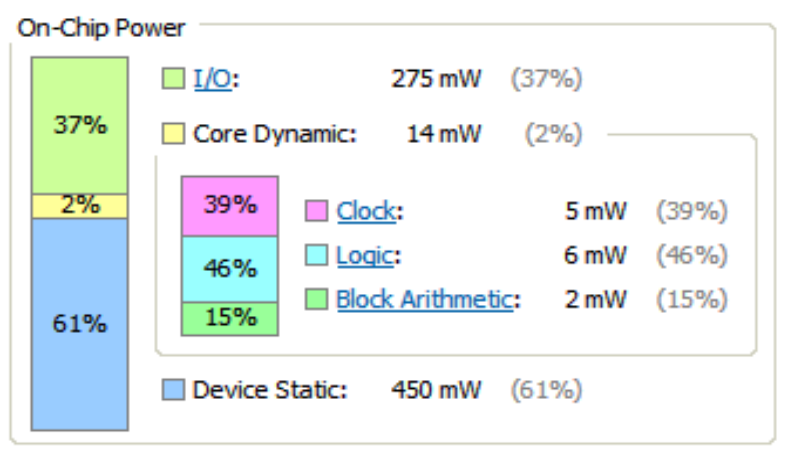

Figure 28. Power Estimation of Transmitter 
From the Figure 28, it is observed that the power consumed by $\mathrm{I} / \mathrm{O}$ is $275 \mathrm{~mW}$, Core Dynamic is $14 \mathrm{~mW}$ and Device Static is $450 \mathrm{~mW}$ in the transmitter.

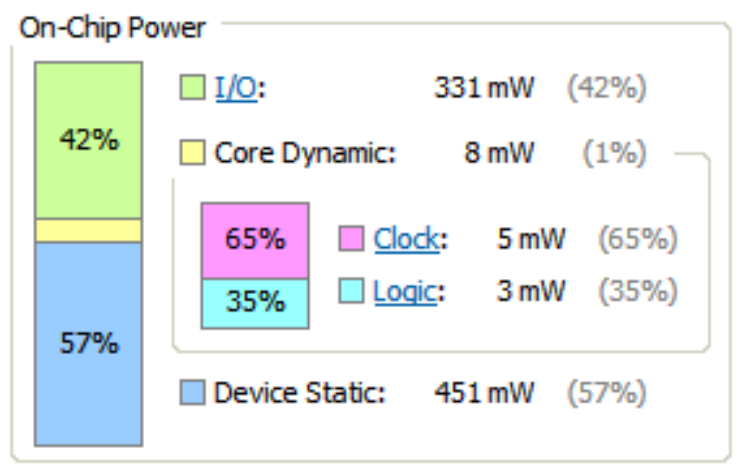

Figure 29. Power Estimation of Channel

From the Figure 29, it is seen that the power consumed by Device Static is $451 \mathrm{~mW}$ in the channel.

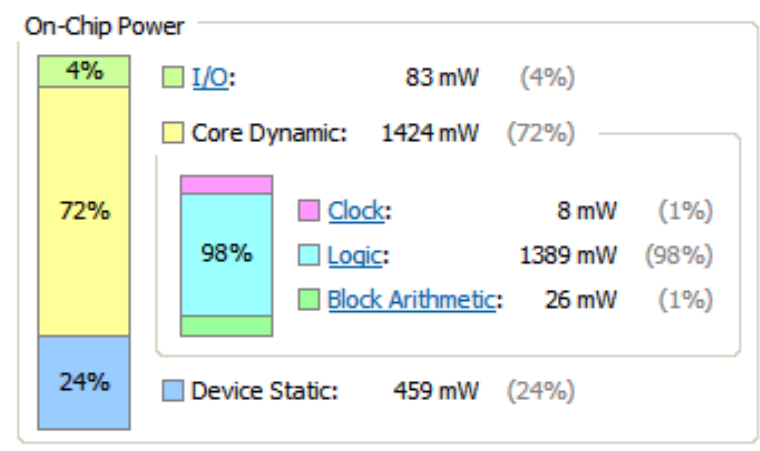

Figure 30. Power Estimation of Receiver

Similarly, from the Figure 30, it is observed that the power consumed by Device Static is $459 \mathrm{~mW}$ in the receiver.

\subsection{Timing Summary}

The timing summary indicates the overall period, input and output times. It is for all clocks and is limited by the slowest path. In Table 1, various timing parameters of SISO and SIMO are summarised.

Table 1. Timing Summary

\begin{tabular}{|l|l|l|}
\hline Parameters & SISO & SIMO \\
\hline Speed grade & -2 & -2 \\
\hline Minimum period & $1.430 \mathrm{~ns}$ & $2.527 \mathrm{~ns}$ \\
\hline Maximum frequency & $699.301 \mathrm{MHz}$ & $395.726 \mathrm{MHz}$ \\
\hline
\end{tabular}

\subsection{Advanced HDL Synthesis Report}

Advanced HDL synthesis report gives the correct technology map and interface to optimize the design. Table 2 and 3 shows the advanced HDL synthesis report of SISO and SIMO. 
International Journal of Wireless \& Mobile Networks (IJWMN) Vol. 6, No. 6, December 2014

Table 2. Advanced HDL Synthesis Report of SISO

\begin{tabular}{|l|c|c|c|}
\hline COMPONENTS & TRANSMITTER & CHANNEL & RECEIVER \\
\hline ROMs & 6 & - & 2 \\
\hline Multipliers & - & 18 & 48 \\
\hline Adders/Subtractors & 1 & 12 & 144 \\
\hline Counters & 1 & - & - \\
\hline Registers & 36 & - & - \\
\hline Latches & - & - & 6 \\
\hline Comparators & - & - & 6 \\
\hline
\end{tabular}

Table 3. Advanced HDL Synthesis Report of SISO

\begin{tabular}{|l|c|c|c|}
\hline COMPONENTS & TRANSMITTER & CHANNEL & RECEIVER \\
\hline ROMs & 6 & - & 2 \\
\hline Multipliers & - & 24 & 96 \\
\hline Adders/Subtractors & 1 & 60 & 288 \\
\hline Counters & 1 & - & - \\
\hline Registers & 36 & - & - \\
\hline Latches & - & - & 6 \\
\hline Comparators & - & - & 6 \\
\hline
\end{tabular}

\section{CONCLuSion}

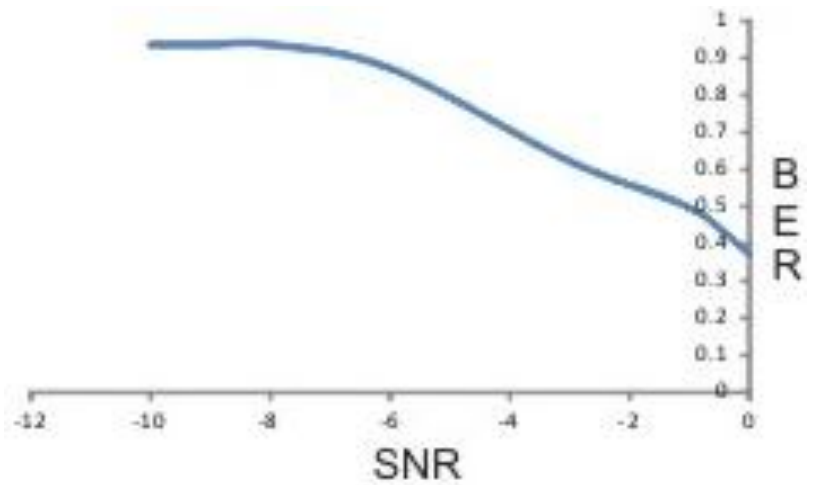

Figure 31. BER Vs. SNR 
The output obtained for varying the variance is shown in the Figure 31. From the figure, it is observed that as the Signal-to-Noise Ratio increases, Bit Error Rate decreases. Similarly, channel emulators can be designed for MISO and MIMO environments under different channels like Rayleigh and Rician fading channels and their performance can be compared.

\title{
ACKNOWLEDGEMENTS
}

I would like to thank God, my family and friends for being with me all the time.

\section{REFERENCES}

[1] S. J. Thiruvengadam and Louay M. A. Jalloul, "Performance Analysis of the 3GPP-LTE Physical Control Channels", Hindawi Publishing Corporation, EURASIP Journal onWireless Communications and Networking, Volume 2010, Article ID 914934, 10 pages.I

[2] Emmanuel Boutillon, Jean-Luc Danger, Adel Ghazel, "Design of high speed AWGN communication channel emulator", IEEE ICECS conference, Kaslik, Lebanon, Dec 2000.

[3] Jean-Luc Danger, Adel Ghazel, Emmanuel Boutillon, Hédi Laamari, "Efficient implementation of Gaussian noise generator for communication channel emulation", 7th IEEE International Conference on Electronicsm Circuits \&Systems (ICECS'2K), Kaslik :Liban (2001).

[4] Hamid Eslami, Sang V. Tran, Ahmed M. Eltawil, "Design and implementation of a scalable channel emulator for wideband MIMO systems", IEEE Transactions on Vehicular Technology, Vol. 58, No. 9, November 2009.

[5] Siavash M.Alamouti, "A Simple Transmit Diversity Technique for Wireless Communications", IEEE Journal on Select Areas in Communications, Vol.16, No.8, Oct,1998.

[6] S. Syed Ameer Abbas, S.J. Thiruvengadam and S. Susithra, "Novel Receiver Architecture for LTEA Downlink Physical Control Format Indicator Channel with Diversity", Hindawi Publishing Corporation, VLSI Design, Vol. 2014, Article ID 825183, 15 pages.

[7] S.S.A. Abbas and S.J. Thiruvengadam, "FPGA implementation of 3GPP-LTE-A physical downlink control channel using diversity techniques", International Journal of Wireless and Mobile Computing, Vol. 9, No. 2, p. 84, 2013.

[8] VLSI Digital Signal Processing Systems: Design and Implementation [book], Keshab K. Parhi.

[9] Digital Signal Processing with Field Programmable Gate Arrays [book], Uwe Meyer-Bäse.

[10] Digital Signal Processing: Principles, Algorithms, And Application, 4/E, John G. Proakis.

\begin{abstract}
Authors
Dr. R. Shantha Selva Kumari is the Professor and Head of the Department of Electronics and Communication Engineering, Mepco Schlenk Engineering College, Sivakasi. She has rich experience in teaching \& research and her specializations are Bio Signal Processing and Digital Communication.
\end{abstract}

Ms. M. Aarti Meena is a PG scholar of Communication Systems in the Department of Electronics and Communication Engineering, Mepco Schlenk Engineering College, Sivakasi. 